MUZIKOLOŠKI ZBORNIK - MUSICOLOGICAL ANNUAL XL

UDK 78:271.5(497.4)"15"

Tomaž Faganel

Musikwissenschaftlischen Institut das Forschungszentrum der Slowenischen Akademie für

Wissenschaft und Künste, Ljubljana

Muzikološki inštitut Znanstvenoraziskovalnega centra Slovenske akademije znanosti

in umetnosti, Ljubljana

\title{
Der Einfluß der slowenischen Jesuiten auf die musikalischen Zustände in Mitteleuropa im 17. Jahrhundert
}

\author{
Prispevek slovenskih jezuitov h glasbeni \\ podobi Srednje Evrope v 17. stoletju
}

ZUSAMMENFASSUNG

Die slowenische kulturelle Auslieferung ist mit den Jesuiten seit ihrer Ansiedlung Ende des 16. Jahrhunderts auf das breitere slowenische Gebiet eng verbunden. Die Einheit des religiösen und kulturellen Wirkens der Gesellschaft in Slowenien formte die einheimische kathlolische Intelligenz und festigte die religiöse Mission der Gesellschaft. In der durchdachten Tätigkeit der Gesellschaft hatte die Musik auch in Slowenien eine besondere Rolle. Die slowenishen Hauptquellen Historia annua Collegii Societatis Jesu Labacensis und Historia Seminarii Labacensis sind auch auf dem Gebiet der Musik mit den Berichten anderer Jesuitenzentren der Provinz vergleichbar. Die Angaben über die musikalische Tätigkeit im Seminar für Musik, die Jesuiten gründeten es vier Jahre nach der Gründung des Collegiums, ermöglichen keinen genauen Einblick in die Musikpraxis der slowenischen Jesuiten. Aus ihr können wir auf die Leistungsfähgikeit und die Besetzungen, die den gewöhnlichen mehsrtimmigen barocken vokal-instrumentalen Partituren entsprechen, auf die Verbindungen mit den Tätigkeiten verschiedenartiger Bruderschaften, Musiktheater und Tanz, Schlüsse ziehen. Die Behauptung, daß die Jesuitem die slowenische Intelligenz und damit
POVZETEK

Slovensko kulturno izročilo je z jezuiti od njihovega prihoda na širše slovensko ozemlje konec 16 . stoletja tesno povezano. Enovito versko in kulturno delovanje Družbe na Slovenskem je sooblikovalo domačo katoliško inteligenco in utrdilo versko poslanstvo Družbe. V njenem domišljenem delovanju je imela glasba tudi na Slovenskem posebno mesto. Glavna slovenska jezuitska vira $\mathrm{Hi}$ storia annua Collegii Labacensis Societatis Jesu in Historia Seminarii Labacensis sta tudi za področje glasbe primerljiva s poročili drugih središč province. Navedbe o glasbenem delu v Glasbenem seminarju, ljubljanski jezuiti so ga ustanovili 4 leta po ustanovitni kolegija, omogočajo umišljeno sliko glasbene prakse slovenskih jezuitov. Ta kaže na zmogljivosti in sestav, ki ustreza običajnim in mnogoglasnim baročnim vokalno-inštrumentalnim partituram, na povezavo $z$ delovanjem raznovrstnih bratovščin, glasbenim gledališčem in plesom. Trditev, da so jezuiti oblikovali slovensko inteligenco in s tem kulturo, je glede na vire nesporna tudi glede na kasnejše delovanje jezuitskih alumnov, od katerih so se mnogi posvetili glasbi. Primer skladatelja p. Dolarja in njegov opus po dokazuje, da je bil razvoj nadpovprečno nadarjenega muzika 
MUZIKOLOŠKI ZBORNIK • MUSICOLOGICAL ANNUAL XL

die Kultur geformt haben, ist den Quellen nach unanfechtbar, auch, wenn wir das spätere Wirken der Absloventen der Jesuitenschule, unter welchen sich viele der Musik widmeten, betrachten. Das Beispiel des Komponisten Pater Dolar und seines Werkes beweist, daß die Entwicklung des überdurschnittlich begabten Musikers im Ordensrahmen möglich und beachtet war, die Ergebnisse seines musikalischen Schaffens waren schon im aktuellen Zeitraum im breiteren Sinn interessant und entsprechend hoch beurteilt. možen in upoštevan tudi v redovnem okviru, rezultati njegovega glasbenega ustvarjanja pa že v aktualnem času $v$ širšem prostoru zanimivi in primerno visoko ovrednoteni.

Die slowenische kulturelle Auslieferung ist mit den Jesuiten seit ihrer Ansiedlung Ende des 16. Jahrhunderts auf das breitere slowenischen Gebiet, damals noch im Rahmen der Habsburger Monarchie, eng verbunden. Das zentrale Kollegium in Ljubljana gründeten die Jseuiten vor 407 Jahren, unweit der Stelle, an der unser Symposium stattfindet. Die Einflüsse und vor allem die Unterstützung der Tätigkeit der Gesellschaft Jesu, verbreiteten sich schon vor Beginn ihrer offiziellen Tätigkeit bei uns, auf den heutigen mitteleuropäischen Raum, bzw. auf den breiteren Habsburger Raum, so war z.B. Textor, ein Bischof in Ljubljana Mitte des 16. Jahrhunderts, Anreger der Gründung des Jesuitenkollegiums in Wien und dessen großzügiger Förderer, die späteren Bischöfe in Ljubljana, Seebach und Tavčar, besonders Letzterer mit Stipendien, haben das Kollegium in Graz, nur um Weniges ältere, als das in Ljubljana, eifrig unterstützt.

Die Einheit des religiösen und kulturellen Wirkens der Gesellschaft auf dem breiteren Gebiet von Ljubljana bzw. des heutigen slowenischen Gebietes, dessen Ziel die Formung und Aufstellung einer einheimischen, slowenischen katholischen Intelligenz war, war das fundamentale Mittel der Durchsetzung und Festigung der religiösen Mission der Gesellschaft in den - heute scheint es - lange andauernden Jahrzehnten der Gegenreformation. In der strategisch durchdachten kulturellen Tätigkeit der Gesellschaft, die sich neben der Schulung vor allem den Künsten mit der Architektur und anderen bildenden Künsten widmete, spielte die Musik eine ausnehmend bedeutende Rolle. Gleich der inhaltlich einheitlich durchdachten Orientierung der Tätigkeit der Gesellschaft Jesu und ihrem allgemeinen Ansatz in allen Läudern der Provinz - in gewissem Sinn können wir ihn als ein unifiziertes Modell ansehen -, können wir auch die musikalische Tätigkeit der slowenischen Jesuiten im 17. Jahrhundert als allgemeines Muster der musikalishe Tätigkeit der Gesellschaft erklären, ohne Rücksicht auf Ort oder Land, auf diesen oder anderen Teil der Provinz. Ein Einblick in die jesuitischen Quellen, die sogenannten Historien des breiteren europäischen Raumes, zeigt ebefalls auf die Einheit der Auffassung der Mission, auf die Einheit des Ansatzes und vor allem auf die Einheit der Diktion des Inhalts. So sind die jesuitischen Hauptquellen aus Ljubljana, die Historia annua Collegii Labacensis Societatis Iesu und die Historia Seminarii Labacensis, durchaus mit den Berichten andere Jesuitenzentren der Provinz vergleichbar.

Die musikalische Tätigkeit wurde vier Jahre nach der Gründung des Kollegiums aufgenommen. Das sogenannte "musikalische Seminar" gründeten die Jesuiten im 
Jahre 1600. ${ }^{1}$ Die Angaben über die Musik in der Hausordnung der Mittelschüler sind sehr spärlich, so ist ein Hinweis auf die musikalische Tätigkeit im Konvikt, vor allem eine gründliche Beschreibung, kaum möglich. Die Rede ist von zwei täglichen Gesangsübungen, eine Viertelstunde nach dem Mittagessen und so auch nach dem Abendmahl, ${ }^{2}$ an anderer Stelle werden die Zöglinge als "musici seminaristae" erwähnt. Die Historia der Gesellschaft gibt an, daß "das Seminar die Musik in der Kirch bestreitet. ${ }^{3}$ Aus dem Jahre 1635 finden wir einen Bericht über einen Kardinalsbesuch, der in schwer verständlicher Diktion anführt: "Im Konvikt wird oft Musik zur Schönheit der Kirche und zu Ehren Gottes gepflegt, Kardinal Palota lobte sie sehr, als er hier nach Rom vorüberreiste. " Die Beurteilung der Kunstgerechtigheit der Musik muß heute leider ausbleiben, da über den Grad der Qualität der Musik in den Berichten nie die Rede ist. ${ }^{5}$

Die Zahl der Jesuitenzöglinge, die sich der Musik widmeten, ist in den Berichten verschieden. Bald nach der Gründung besuchen das Seminar 60 Zöglinge, im Jahre 1617 sind es schon hundert, wobei berechtigt bezweifelt werden muß, daß es sich bei allen Alumnen ausschliesslich um Musiker handelt. Eine Hundertschaft aktiv studierender Musiker wäre ein wahrer Luxus auch für Ordenshäuser großer und größter Städte, z.B. in Wien. Der Bericht aus Ljubljana ${ }^{6}$ teilt die Zöglinge auf Stipendienempfänger, die sich obligat der Musik widmen müssen, und auf gewöhnliche, "convictores", die entweder finanziell gutstehend und als solche aristokratischer Herkunft, oder ohne jeglicher musikalischer Begabung waren. Man kann mit dem Grazer Fernandeum eine Paralelle ziehen, im 17. Jahrhundert kennt es eine ähnliche Teilung der Zöglinge und auch mit dem Wiener Ordenshaus der Heiligen Ingantius'und Pankratius', natürlich handelte es sich hier um ein zentrales Ordenshaus mit Privilegien, zusammengeführt wurden in erster Reihe bagabte Musiker für die kirchenmusikalische Praxis und die Theatertätigkeit.

In der Mitte des 17. Jahrhunderts berichtet die Seminarchronik aus Ljubljana erstmals Näheres über die Musik. Der Verfasser zählt 4 Posaunen, 4 Geigen - "neue", 2 Bässe, verschiedenartiges Notenmaterial auf, man spielte auf eine Trombe marina, "mit Hilfe welcher man sich in angenehmen Harmonien versuchte. Am Rande sei bemerkt, daß die Trombe marina bzw. die sogenannte Nonnentrompette auf zeitgenössischen Fresken auch vom wandernden Tod gespielt wird. Von diesem allegorischen Gebrauch des Instruments ist es nicht weit zur Musik der Bußgottesdienste, welchen sich die Jesuiten in ihrer regulären Tätigkeit und in religiösen Aktivitäten verschiedener Bruderschaften, ausgiebig widmeten. Doch darüber später.

${ }^{1}$ Vgl. Historia Seminarii Labacensis, in qua origo, progressus, benefactores, eiusden alumni continentur. Ad anno $M D C$, Ms 156, Handschriftensammlung, National- und Universitätsbibliothek, Ljubljana.

2 Vgl. Liber archivum Labacensis, 1717, S. 594 und 597-599.

3 "[...] Seminarium cursum suum tenuit templum musica [... ] "Vgl. Historia annua Collegii Labacensis Societatis Jesu Labacensis, Handschriftensammlung, 180 r, Archiv der Republik Slowenien, Ljubljana.

4 "Ad ornatum templi Deique honorem, quam et ipse cardinalis Palota, hac transiens Romam vehementur dilaudavit et studia Societatis tum in seminario tum in scholis approbavit."Prim. Historia annua Collegii Societatis Jesu Labacensis, [143], Ljubljana, 2002, 117.

5 excuisita, auch rara musica.

${ }^{6}$ Für den Zeitraum von 1662 bis 1645 gibt es keine ausfürliche Listen. 
Die musikalischen Pflichten der Zöglinge werden nur ausnahmsweise erwähnt. Im Jahre 1654 lesen wir über 2 Organisten, 5 Sopranisten, 1 Geiger, 1 Tubicenisten (heute verstehen wir unter diesem Begriff jemanden, der ein Blasinstrument spielt), 1 Tubisten (wahrscheinlich ein Musiker der auf ein tiefes Blasinstrument spielt) und 13 gewöhnliche "musices". Der Bericht erwähnt auch einen musikalischen Leiter. An anderer Stelle lesen wir nach nicht ganz 30 Jahren, von einem Sopranisten und Geiger, der nach dem Stimmbruch zum Tenoristen "avanciert" ist.

Wenn wir die Anführungen und Angaben halbwegs zu ordnen versuchen und die Berichte aus den Gründungsjahren der Jseuiten mit denen aus der zweiten Hälfte des Jahrhunderts vergleichen, können wir feststellen, daß viele Zöglinge zugleich gesungen und auf ein Instrument gespielt haben. Man findet auch solche, die mehrere Instrumente beherrschten, ein Saiten- und Holzblas- oder ein Blasinstrument, in jenen Zeiten war dies unter Musikern keine Seltenheit. Als fiktives Gesamtbild der Ausführenden können wir uns ein Ensemble vorstellen, welches den Aufführungen gewöhnlicher, aber auch merhrstimmiger barocker vokal-instrumentaler Partituren entsprach. Man kann eine Gruppe zusammenstellen die mit Veränderungen bis zu 10 Sänger, bis zu 8 Geiger, 1 Violisten, 2 Bläser, ${ }^{7}$ bis zu 4 Posaunisten und 1 Organisten zählte.

Auf anderer Seite sind die Berichte über die Musik in der Historia der Gesellschaft wesentlich allgemeiner, mit Rücksicht auf die Allgemeinheit des Jahresberichtes ist das auch verständlich. Es gibt Angaben darüber, wann der eine oder andere Zelebrant am Vorfeiertagsabend die Vesper "sang", am Feiertag die Messe oder die Litanaien "sang". Dazwischen gibt es freilich auch Stellen, die detaillierter über die musikalische Praxis und ihren Inhalt berichten. Schon im Jahre 1606 z.B. lesen wir, daß die "Knaben die Weihnachten mit lieblichem Gesnag und dem Vortrag slowenischer Lieder so wie auch instrumentaler Musik feierten." Vier Jahre später erfahren wir, daß "der Abt aus Sticna der Kongregation und dem Kollegium ein höchst geeignetes Instrument für unsere Chor, allgemein als Regal bekannt, ausgehändigt hat. Er hatte dafür 200 Gulden gezahlt." Zehn Jahre später lesen wir darüber, daß der "Freibaron Markvard Egg unserer Kirche eine große 200 Gulden werte Orgel dagebracht hatte." ${ }^{10}$ Den wahren Wert der Orgel wollen wir hier nicht beurteilen, für beide Instrumente führt der Chronist den gleichen Preis, 200 Gulden, an.

Die musikalische Tätigkeit ist auch in den ersten Jahrzehnten des 18. Jahrhunderts dokumentiert, in einem Zeitraum also, in welchem, so scheint es, die musikali-

7 Wahrscheinlich waren sie Clarinisten bzw. Kornettisten. Die Ausführenden auf diesen Instrumenten nahmen wegen des schwierigen Spielens auf die Instrumente in der Hierarchie der damaligen Musiker eine besondere Stellung ein, so den Pflichten, wie den Einkommen nach. Aus den Benachrichtigungen der Jesuiten in Ljubljana ist Ähnliches nicht zu entnehmen.

8 "Venusta carminum Slavicorum recitatione et cantum varietate nec non symphonia musica infantem Christum pueri in die natalis Domini concelebrarunt."Vgl. Historia annua Collegii Societatis Jesu Labacensis, [50], Ljubljana, 2002, 53.

9 "Reverendissimus item abbas Siticensis congregationi et huic collegio obtulit instrumentum musicum, vulgo regale dictum, nostro choro percommodum, pro quo ducentos florenos exposuerat."Vgl. Historia annua Collegii Societatis Jesu Labacensis, [68], Ljubljana, 2002, 66.

10 "[... ] generosus dominus Marquardus ab Egg, libero baro, ordinis Teutonici commendator, qui organum maximum, quod pretium 200 florenorum excedit, templo nostro donavit."Vgl. Historia annua Collegii Societatis Jesu Labacensis, [84], Ljubljana, 2002, 78. 
sche Aktivität und die künstlerische Rolle der Gesellschaft durch die Entstehung der musikalischen Institutionen und Vereinigungen allmählich nachzulassen begannen. Das Instrumentarium verbreitete sich und entsprach zeitgenössischen Bedürfnissen. Es werden Namen von Oboisten, Fagottisten genannt, es erscheint ein, für diesen Raum neues Baßstreichinstrument - das Violoncello. Für den musikalischen Leiter werden aktuellere Titel gefunden, aus denen man entnehmen kann, daß sie nicht mehr nur an ein Haus oder an eine Ordenshiearchie gebunden waren: director musices, compositor. Die Benennung regens chori finden wir in der Historia im Jahre 1672 für den Pater Ignac Kumeš, der zwar unserer Musikwissenschaft nicht bekannt ist. ${ }^{11}$ Später, in den Jahren 1687 und 1689 erwähnt der Chronist noch m. Karl Stadler und $\mathrm{m}$. Janez Haas, beide mit dem Titel praefectus chori. ${ }^{12}$

Nach dem Vorbild der Leitungsstrukturen anderer Jesuitenhäuser und Institutionen war auch in Ljubljana einer der Präfekte auch praefectus musicae, der für die Musik Sorge trug. Die Historia berichtet über den bedeutendsten und heute bekanntesten Musiker der Jesuiten slowenischer Herkunft, Pater Janez Dolar, als Musiker nur zwei Mal: im Jahre 1657 als er als scholarum simul et musicae praefectus das feierliche Ordensgelübde abgelegt hatte und Profeß wurde ${ }^{13}$ und ein Jahr später, als er als scholarum et diesmal chori praefectus nach Passau gesandt wurde. ${ }^{14}$ Es besteht kein Zweiel darüber, daß er an allen Orten seines Ordensdienstes von Ljubljana, über Steyr, Wien, Graz, Judenburg, wieder Ljubljana, danach Passau, Gyõr musikalisch tätig war, nicht nur als Leiter der Musik, sondern auch als Komponist. In seiner Todesanzeige in den Nachrichten des Wiener Ordenshauses, dessen "historicus" er selbst bis zu seinem Tode war, steht geschrieben, daß er "um ihre [nämlich der Musik] Ehre zu vergrößern, selbst heilige Lieder komponierte, die nicht nur in Hinsicht auf die künstlerischen Gesetze ausgezeichnet waren, sonder haben auch den Sinn und die Empfindungen des Textes ausgedrückt." ${ }^{15}$ Und in der Fortsetzung: "In dieser Kunst machte er solche Fortschritte, daß es nach allgemeiner Meinung vieler musikalische Fachleute nur wenig solche gab, die sich mit ihm hätten messen können; deswegen versuchten seine heiligen Lieder nicht nur unsere Häuser zu erwerben, um sie konkurrierten auch viele Kloster anderer Orden im Lande und auch anderswo." ${ }^{16}$ Diesen Hinweis bestätigen vor allem auch die Angaben über seine Werke in den Verzeichnissen der Musikarchive in erster Reihe in Kremsmünster, auch in Šentpavel/St. Paul in Kärnten, die Erwähnung seiner Werke in der Tschechei und in Thüringen und vor allem in Mähren, wo die Zeit einen Teil seiner Kompositionen auch vor dem völligen Vergessen behütete.

${ }^{11}$ "M. Ignatius Kumesch, principista, regens chori, praefectus hospitum, visitator orationis secundae." Vgl. Historia annua Collegii Societatis Jesu Labacensis, [396], Ljubljana, 2002, 263.

12 "M. Joannes Haas, poeta, praefectus chori."Vgl. Historia annua Collegii Societatis Jesu Labacensis, [508], Ljubljana, 2002, 358.

${ }^{13}$ Vgl. Historia annua Collegii Societatis Jesu Labacensis, [310], Ljubljana, 2002, 197.

${ }_{15}^{14}$ Vgl. Historia annua Collegii Societatis Jesu Labacensis, [314], Ljubljana, 2002, 202.

${ }_{15}$ An dieser Stelle enthalte ich mich, im Sinne der barocken Afektenlehre, bewußt jeglichen Kommentars.

${ }^{16} \mathrm{Vgl}$. Nekrolog in Litterae annuae Provinciae Austriae S. J. Anni 1673, Österreichische Nationalbibliothek, Wien, Cod. 12070, 9-10. 
MUZIKOLOŠKI ZBORNIK • MUSICOLOGICAL ANNUAL XL

Wie aus den Nachrichten des Seminars in Ljubljana zu entnehmen ist, wechselten die Musikpräfekte häufig ab, ähnliche Verhältnisse finden wir in den Historien überall auf dem breiteren Gebiet der Habsurger. Eine Beurteilung des musikalischen Vermögens einzelner Leiter und ihres Einflußes auf die Entfaltung der musikalischen Tätigkeiten, ist heute leider nicht möglich. Bestimmt aber verfügte das Ordenshaus in Ljubljana nicht über so viele Präfekte - gute Musiker, wie Ordensleute, die als Leitende des Institutes einer dem anderen folgten. Den Angaben nach mussten die sich abwechselnden Leiter in Ljubljana offensichtlich nicht immer gründlich der Musik widmen. Anders waren z.B. die Verhältnisse im Seminar der heiligen Ignatius' und Pankratius' in Wien, wo Pater Janez Krstnik Dolar, ein Jesuit aus Kamnik, als Regens 11 Jahre, bis zu seinem Tode 1673 wirkte. Seine verschiedenartige musikalische Obligationen sind näher in der Todesanzeige in den Wiener Jahresbüchern beschrieben. ${ }^{17}$ Seine Verpflichtungen in Wien übertrafen weit die der Regenten in Ljubljana, neben der Arbeit im Seminar mußte er auch die anstrengende musikalische Leitung in der Kirche und im Theater Am Hof bewältigen.

Mit der musikalischen Praxis der Jesuiten sind auch die entstehenden Bruderschaften verbunden. Die Kongergation der Himmelfahrt Mariä erwähnt der Chronist erstmalst im Jahre 1605 und als elementare Aktivität der Bruderschaft Prozessionen mit Musik. Eine klare inhaltliche Grenze zwischen einer feiertäglichen Prozession und einer Buß- und Dankesveranstaltung, einer s.g. suplicatio zu ziehen, ist heute unmöglich. Das Mitwirken der Musiker ist in den Berichten bestätigt, obwohl nicht genau. Wurde gesungen, gespielt, auch getanzt? Einen Teil der Antwort finden wir in der Angabe, daß die Bruderschaften vor allem von Musikern geleitet wurden, z.B. vom Dalmatiner Georgicenus - Grgičević , dem Triestiner Gobb, musicae praefectus Skubezz - wahrscheinlich Skubic, und nicht zuletzt von Pater Ioannes Dolar und später von Mihael Omerza. Mit allem Prunk wurde im Jahre 1640 eine supplicatio zu Ehren des Gründers und der Schutzheiligen der Bruderschaft, der in den Himmel aufgenommenen Maria mit einem Fackelzug und einer Regatta auf dem Fluß Ljubljanica vorbereitet, "unter feierlichem Dröhnen der Bombarden [Baßschalmeien bzw. Pommern] gleitete das Schiff ruhig zum Landeplatz." ${ }^{18}$ Am Rande sei als Bemerkung erwähnt, daß es anlässlich dieser Feierlichkeit zu einem Brand kam: wegen der Geschosse von der Burg und den Ufern des Flußes aus, entflammte auf einem Frachtboot mit Heu ein Feuer - die Regatta wurde nämlich, so der Chronist, von "militärischen Klängen"begleitet - die Musik "schlug in Trauer um, das Lob in Verhöhnung, durch die Gassen war zu hören 'Jesuiten, Brandstifter, Kanaillen' und was immer schon das böse Volk auszuspeien vermochte." 19

Obwohl unsere Kenntnisse über die Theatervorstellungen der Jesuiten abgerundet sind, wissen wir über die Musik bei diesen Veranstaltungen wenig. Die zur Verfügung stehenden Synopsen weisen darauf hin, das die allegorischen Teile der Spiele vertont bzw. gesungen waren. Zu finden sind Ausdrücke wie praeludium musicum,

\footnotetext{
${ }^{17}$ Vgl. Litterae annuae Provinciae Austriae S. J. Anni 1673, Österreichische Nationalbibliothek, Wien, Cod. 12070, 9-10.

${ }^{18}$ Vgl. Historia annua Collegii Societatis Jesu Labacensis, [165], Ljubljana, 2002, 127.

${ }^{19}$ Vgl. Historia annua Collegii Societatis Jesu Labacensis, [165], Ljubljana, 2002, 127.
} 
inter- und postludium. Unter den nomina actorum ist auch ein chorus musicus erwähnt. Oft wird vom Chronisten der Ausdruck dialogo oder rapresentatione niedergeschrieben, beides sind eigentlich Fachausdrücke des frühen Musiktheaters, doch erklärt er, außer des Titels, nie näher die Art der Ausführung. Die Musik war also nicht eine zufällige Einlage, nur eine Randkulisse, sondern war ein selbstständiger Teil der musiktheatralischen Gänze. Die Überlegungen über die Inszenierung der Musik auf der Bühne erlaubt, oder fördert sogar eine Anführung im älteren slowenischen historischem Schrifttum - ein Chronist berichtet über eine heute verlorene gedruckte Sammlung von Pater Dolar Drammata seu Miserere mei Deus. ${ }^{20}$ Die Sammlung kann zweifelsohne mit den charakteristischen Andachten der Jesuiten anlässlich der Schlußfeierlichkeiten der Karwoche verbunden werden, auf die Ehrung des heiligen Grabes am Karfreitag, an das sepolcro, das oft vom Chornisten der Familie in Ljubljana ewähnt wird, obwohl selten mit diesem Ausdruck. Eben dieses sepolcro wurde regelmäßig in der Kaiserkapelle in Wien inszeniert - in diesem Kontext möchte ich auf das kompositorische Opus des Kaisers Leopold hinweisen - und auch auf breiterem Gebiet der österreichischen Länder im allgemeinen. ${ }^{21}$

In mehreren Fragmenten wird festgestellt, daß die Spiele mit Musik- und Tanzeinlagen, die ihr Bestandteil waren, ausgestattet wurden: vor allem mit battaglien und in apollinischen Sequenzen, die sich auf die Titel der Spiele bezogen, auch mit pastoraler Musik. Im Verzeichnis der Mitwirkenden lesen wir von saltatores, sogar vom saltum magister, was in die Sprache der Musik übersetzt, selbstständige instrumentale Musik bedeutet. Hier verwischt sich die Grenze zwischen Prozession, Passionsspiel, der supplicatio un dem sepolcrum gänzlich und enndgültig. Es scheint, daß wir gänzlich an die momentane Diktion der Chronisten angewiesen sind, die die Musik allzu selbstverständlich erwähnen, auch zu allgemein und am Rande, zu wenig beschreibend und vor allem unregelmäßig.

Bei der Einordnung der Erwähnungen der Musik in den slowenischen Jesuitenchroniken in den breiteren Raum, müssen wir auch die geografischen Angaben in Betracht ziehen. Neben den zentralen Ordenshäusern berichten die Verfasser auch über das Geschehen auf den zugehörigen Seitenbesitztümern, der geografische Raum aber, der in den Chroniken nicht nur in personalen Anführungen beschrieben wird, ist für die Verhhältnisse vor vier Jahrhunderten wahrhaft imposant: Varaždin, Rijeka, Zagreb, Klagenfurt, Graz, Leoben, Judenburg, Linz, Wien, Trnava, Bratislava, Gorizia, Triest, Venedig, Loreto, Prag, Český Krumlov, Olomouc, Passau und auch Antwerpen.

Die anfangs gestellte Behauptung, daß die Jesuiten die slowenische Intelligenz und dadurch die Kultur geformt haben, bestätigt sich als umunstritten auch, wenn wir das spätere musikalische Wirken der Absolventen der Jesuitenschulen betrachten. Viele unter ihnen widmeten sich der Musik, wobei sie durch ihren priesterlichen

\footnotetext{
${ }^{20}$ Vgl. Viktor Steska, Dolničarjeva 'Bibliotheca Labacensis publica', in: Izvestja muzejskega društva za Kranjsko 10 (1900), 145.

${ }^{21}$ Beim Sepulcrum wurden beim Heiligen Grabe Psalmen gesungen, es wurden Passions- und andere Bußfragmente vorgeführt. Im Kollegium in Ljuljana wurde 1634 „ad sepulcrum “ die Szene Maria und Magdalena in deutscher Sprache bzw. in „versu germanico“ ausgeführt. Vgl. Historia annua Collegii Societatis Jesu Labacensis, [141], Ljubljana, 2002, 116.
} 
MUZIKOLOŠKI ZBORNIK - MUSICOLOGICAL ANNUAL XL

Grundberuf und gelegentlich auch durch den Rahmen des Ordenslebens nicht behindert wurden. Ähnlich wie in anderen mitteleuropäischen Städten finden wir die Jesuitenschüler vor allem als Musiker in den städtischen Kirchen und als Stadtmusikanten vor. Obwohl wir heute auf Grund der Berichte ihre Fähigkeiten nicht bemeßen können, zeugt die Verzweigtheit ihrer Tätigkeiten davon, daß viele von ihnen bestimmt die zunfthandwerklichen Fachkenntnisse überschritten. Das Beispiel des Komponisten Pater Dolar und seines erhaltenen Werkes beweist, daß die Entwicklung eines überdurchschnittlich begabten Musikers möglich und beachtet war, auch im Ordensrahmen, die Ergebnisse seines musikalischen Schaffens waren schon im aktuellen Zeitraum im breiteren Sinn interessant und entsprechend hoch beurteilt. 